\title{
REMOTE SENSING AND CITY INFORMATION MODELING FOR REVEALING THE COMPLEXITY OF HISTORICAL CENTERS
}

\author{
Federico Mario La Russa ${ }^{1}$, Mariateresa Galizia ${ }^{1}$, Cettina Santagati ${ }^{1}$ \\ ${ }^{1}$ Department of Civil Engineering and Architecture, Università degli Studi di Catania, Catania, Italy \\ federico.larussa@phd.unict.it, cettina.santagati@unict.it,mgalizia@dau.unict.it
}

KEY WORDS: City Information Modeling, Expeditious Urban Survey, SLAM, TLS, Photogrammetry, Historical Centers, GIS, HBIM

\begin{abstract}
:
Historical centers represent the outcome of transformations and stratifications of the cities across the centuries. The knowledge of a historical urban environment requires an analytical methodology articulated on several interconnected levels of investigation to model a multi-layered complexity that encompasses the geometric and stylistic features of places (blocks irregularities, narrow streets, stratified buildings), the accessibility (pedestrial zone, no flyzone), the use of existing data (GIS, cartographies). Today the challenge for historical centers is dual: on the one side to make use of expeditious technologies to acquire data, on the other one to create 3D city models that allow to manage, visualize, enquire and use these data in a unique digital ecosystem. Our research deals with a multi-sensor data acquisition, evaluation and integration with the aim of creating informed and responsive 3D city models (CIM) that constitute a synthesis of the survey conducted and become the support for simulations in various contexts (seismic risk, hydraulic, energy performance).
\end{abstract}

\section{INTRODUCTION}

Historical centers represent the memory of the choices that have drawn the history of a place; that is, the outcome of transformations and stratifications of the cities across the centuries. The knowledge of a historical urban environment requires an analytical methodology articulated on several interconnected levels of investigation to model a multi-layered complexity that encompasses the geometric and stylistic features of places (blocks irregularities, narrow streets, stratified buildings), the accessibility (pedestrial zone, no flyzone), the use of existing data (GIS, cartographies). Urban survey and 3D modeling play a pivotal role in the various phases of the cognitive process. Indeed, the interpretation of the collected geometric-spatial data together with the critical reading of historical documents (i. e. cadastral maps, archive sources) enable us to understand the link between the traces of the past and the present. Today the challenge for historical centers is dual: on one side, to make use of digital technologies to acquire data, on the other one to create systems that allow to manage, visualize, enquire and use (i. e. for simulation purposes) these data in a unique digital ecosystem. Moreover, the anthropic and natural hazards (earthquakes, flows, etc) to which historical centers are prone require a continuous monitoring of their state of conservation, necessarily sustainable in time and resources. In this direction, the use of expeditious techniques such as MMS (Mobile Mapping Systems) would support wide campaigns for mapping historical centers. However, the narrowness and accessibility difficulties create obstacles towards the use of vehicle-mounted MMS. In addition, the use of flying drones in these areas often is limited not only for the legal and privacy restrictions, but also for the presence of obstacles such as antennas, wires, balconies, ect.

In this sense, a first research question arises: is it possible to use fast mapping urban mapping techniques such as spherical photogrammetry or iMMS (indoor Mobile Mapping Systems) based on SLAM (Simultaneous Localization and Mapping) technologies? Is the level of accuracy and quality of the data sufficient for the requirements of urban surveying?

Another critical issue is related to the data obtained: how to integrate multi-source and multi-scala data for the creation of a City Information Modeling?

Our work focus on these research questions. We investigate the use of the brand-new iMMS BLK2GO Leica's instrument against GOPRO Fusion 360 and we test the possibility to extract from Google Earth 3D mesh tiles of the buildings. The aim is the creation informed and responsive 3D city models (City Information Modeling - CIM) that constitute a synthesis of the survey conducted and become the support for simulations in various contexts (seismic risk, hydraulic, energy performance) (Bocconcino et al, 2021).

As case study we chose an emblematic area of the city of Catania (Italy), the ex "extra moenia" district of Rinazzo (made up of narrow, twisting streets and one/two-floor buildings) which in 1881 was affected by the opening of a new road. This intervention has dismembered the area into two parts. The city blocks include different morphological characteristics (urban canyon ratios) and building typologies, that range from highlevel residential buildings (palatial houses) to spontaneous buildings (one- or two-storey cells), which are still characterised by socio-cultural degradation.

\section{RELATED WORKS}

\subsection{Urban Survey}

The city is a complex evolutive system that adapts to the history of places and also is interpreted and modified by successive generations. In the evolution of historicized urban centers, there are many repeated constants and many variables, consisting mostly of new readings of places, new interpretative approaches that represent the change that allows the system to adapt. In this contest, the role of urban survey, as highlighted by Dino Coppo, is to identify, highlight, recognize, relate, describe and represent, the set of values present in the investigated area with 
the aim to build the formal and structural invariants present in the construction of the image of a city (Coppo, Boido, 2010). In this perspective, urban survey can be envisioned as a multilayered knowledge system, open and implementable over time. Nowadays, digital tools give a precious support to the investigation on complex urban contexts and processes, generally characterized by articulate relationships between multiple aspects (Boido et al, 2021; Galizia, Santagati, 2012). Moreover, the fragility of our historical city centers requires specific methodologies that respond to the need to have a rapid mapping of the investigated site (Predari et al, 2019) and/or models for the simulation or the management of critical scenarios (Bocconcino et al, 2021; La Russa, Santagati 2020).

\subsection{GIS/BIM/CIM}

In recent years there have been a significant number of contributions that attempt to describe CIM in accordance with information modelling standards. It is therefore necessary to identify which characteristics allow defining a CIM model as opposed to a 3D GIS or a BIM extended to the urban scale. According to Xue, F. et al. (2021), the meaning of the 'I' in 'Information' in CIM provides the correct key. The first works where CIM was mentioned concern the alignment between point clouds and georeferenced 3D models in a GIS environment (Julin et al, 2018). In a GIS information is managed by overlapping layers within 2D geometries ( $\mathrm{Lu}$ et al, 2018), whilst CIM considers data from urban analyses (such as pedestrian flows, energy simulations, construction techniques) that are related to each other through relationships (similarly to what happens in BIM) and not only by adjacency of georeferenced positions. A semantically defined 3D model developed within a GIS software can therefore be considered a specific subcategory of CIM (Liu et al, 2017).

As regards BIM, it concerns all the information at the architectural scale functional for the design, management and conservation of a building. In a CIM, on the contrary, the relations between the single architectural components are directly connected to the information related to the threedimensional urban context.

The integration of $(\mathrm{H}) \mathrm{BIM}-\mathrm{GIS}$ data towards the creation of a CIM for the management of historical heritage in city centers is an increasing need. Several research efforts have been done in this direction (Bonfanti et al, 2021; Vacca et al, 2018; Bocconcino et al, 2021; La Russa, Santagati et al, 2020) although this is still an open research topic.

\subsection{Urban mapping fast acquisition techniques}

The last years have seen a growing development of technologies and sensors for fast 3D acquisitions which allow expeditious mapping campaigns in very complex environments. The fastest solution for mapping the city can be envisaged in vehiclemounted MMS, although they are not very suitable in presence of high-density historical urban centers. Indeed, they are characterized by pedestrian and narrow streets, buildings of different heights and varied street ratios (Barrera-Vera, Benavioles-Lopez, 2018). In these cases, it is preferable to adopt other solutions such as spherical photogrammetry or iMMS tools. The latter is based on SLAM algorithms that allow to capture $3 \mathrm{D}$ point clouds in real time by walking in the area of interest. The drawback is related to the low levels of density and accuracy of these sensors (compared to TLS solutions), as well as the drift errors along the trajectory that may affect the global accuracy. These new devices are constantly evolving technologies that need to be assessed against more consolidated techniques such as photogrammetry and TLS. In literature we find different approaches to evaluate iMMS data both in indoor and in outdoor contexts (Nocerino et al, 2017; Sammartano, Spanò, 2018; Chiabrando et al, 2018; Sammartano et al, 2021; Salgues et al, 2020).

As regards spherical photogrammetry, in their studies Abate et al (2017) and Barazzetti et al (2018) demonstrate that accurate metric reconstructions can be achieved using low-cost sensors. In addition, Teppati Losè et al (2021) verify in their work the integration between iMMS and spherical photogrammetry for the survey of the Montanaro bell tower.

Since the Blk2go is new in the market, few studies about its performances are currently available. In particular, one is addressed at the evaluation of its usability for the generation of DTM in highly vegetated area for detecting and documenting archaeological anomalies (Limongiello et al, 2020); another one concerns narrow spaces such as indoor corridors or multi-store buildings (Piniotis et al, 2020). In both cases the outcomes of the evaluation return an accuracy that represents scales of 1:100 or 1:200, fully compatible with the aim of this research work.

\section{METHODS AND TOOLS}

\subsection{Methods}

The first step aimed at the investigation of the historical centers foresees a knowledge phase with the collection of historical documentary material (cadastral maps, archive projects, cartographies) useful for the reconstruction of the evolution process. These data have to be integrated with existing geodata available from open providers (Open Street Map), local authorities or academic studies in order to have the basis for the first development of a CIM in accordance with CityGML standards (LOD 0-2). A campaign of 3D acquisition then is required to refine the CIM and describe the features of the building (LOD 3-4). In this sense, it is preferable to use expeditious urban-scale survey techniques. Considering the continuous development of the available technologies an assessment is required in terms of accuracy and detail and integration between different technologies for the subsequent 3D modeling. This latter, will be carried out according H-BIM protocols adapted to the urban scale requirements: definition of the Level of Information Needed; classification of architectural elements present in the urban scene; modeling of the envelope elements and facades; informative enrichment of the architectural elements of the model.

In this work we focus on the experimental field survey phase with the comparison of different techniques (TLS, spherical photogrammetry, SLAM) in order to evaluate their efficacy and the possibility of integration in similar urban configurations. The use of UAVs has not been contemplated in order to simulate contexts with no-fly zones or where it is impossible to fly the drone at sight due to the volumetric complexity of the blocks considered. In addition, we will also develop a LOD 2 CIM model of the city block under examination through the use of Grasshopper (Visual Programming Language inside Rhinoceros) which provides a common environment for datafusion operations.

\subsection{Tools}

The BLK2GO is a small dual axis LiDAR. Its core technology the GrandSlam - is based partially on the SLAM technology, simultaneously combining high-speed dual axis LiDAR, Multicamera Vision System (MVS) and an inertial measurement unit that makes the instrument selfnavigating. The MVS include an integrated camera plus three panoramic cameras for visual navigation via SLAM. The generated point 
cloud has RGB attribute. The BLK2GO Live app allows to view essential data while scanning is in progress. To improve selfregistration during data acquisition it is preferable to define closed paths that start and end by the same "docking base". This is useful to prevent and detect drift errors, optimize cloud registration and reduce alignment error. Table 1 summarizes the specifications of the instrument.

\begin{tabular}{|l|c|}
\hline Specifics & BLK2GO \\
\hline Dimensions & $28 \times 8 \mathrm{~cm}$ \\
Weight & $775 \mathrm{gr}$ \\
Points/second & 420.000 \\
Field of view & $360^{\circ}$ (horiz.) $/ 270^{\circ}$ (vert.) \\
Acquisition range & $0.5-25 \mathrm{~m}$ \\
Relative accuracy & $6-15 \mathrm{~mm}$ \\
Absolute position accuracy indoor & $20 \mathrm{~mm}$ \\
High Resolution Camera & 12 megapixel $\left(90^{\circ} \times 120^{\circ}\right)$ \\
Panoramic vision system & 3-camera system, 4.8 \\
& Mpixel $300^{\circ} \times 135^{\circ}$ \\
\hline
\end{tabular}

Table 1. BLK2GO main specifications

For SFM acquisitions we used a GOPRO Fusion 360 action camera. It has a CMOS $1 / 2.3$ " sensor with two 9 Megapixel lenses with a $180^{\circ}$ angle of view which permit the acquisition of spherical photos and immersive videos. Its small size $(74 \mathrm{x} 75 \mathrm{x}$ $40 \mathrm{~mm})$ and light weight (220 g) make it excellent for moving around city blocks.

\section{CASE STUDY}

The case study under examination deals with a spontaneous building fabric of the historical center of Catania the Santa Caterina al Rinazzo district, which is located in the north-east area of the eighteenth-century extra-moenia expansion of the city.

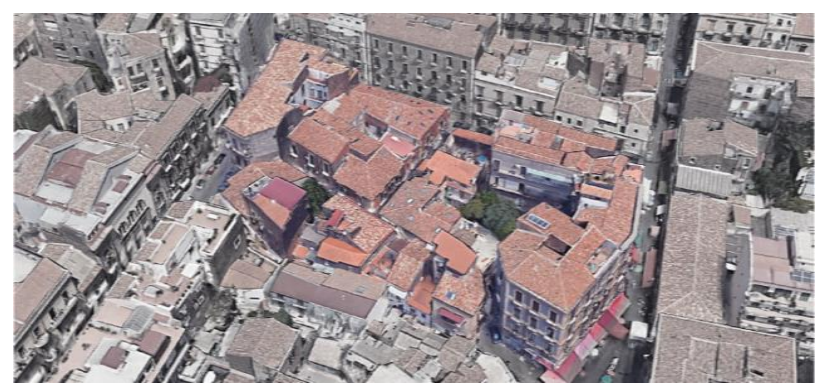

Figure 1. Urban area examined in the case study

In 1881 it was decided the tracing of a new road axis, located close to the entrance to the Public Villa on the main street (Via Etnea) and directed toward the sea. The construction of the new Via Umberto I will strongly break the continuity of the existing building tissue, interrupting the morphological unity of the site. The superimposition of the new road axis will dismember the spaces since it will lead to the demolition of parts or entire buildings and the plano-altimetric reconnection of the involved urban environment.

The new structure will worsen the sanitary conditions of the place, so as to charge in 1888 the engineer Gentile Cusa for the drafting of the "Master Plan of rehabilitation and expansion" of Catania (Gentile Cusa, 1888). The plan foresees the opening of Via Mangano (now Via Filippo Corridoni) and the new Via Santa Caterina, never built, in order to ventilate the blocks often below street level. As a matter of fact, behind the new nineteenth-century road axis characterized by the scenes of the palaces of the new middle class, narrow alleys and courtyards, legacy of the Mediterranean culture, find space in the micro building tissue (Restuccia et al, 2011). The district originally developed at different heights and had non-homogeneous blocks with shapeless lots, narrow courtyards and streets of penetration. This determined a poor ventilation in addition to health and hygiene problems caused by the lack of networks of rainwater drainage. The block examined is part of this urban renewal and is located near Piazza Carlo Alberto, historic seat of the municipal market called "Fera u Luni", and defined by a tract of the ancient via Santa Caterina, via Spampinato and via Grotte Bianche (figg. 2, 3).

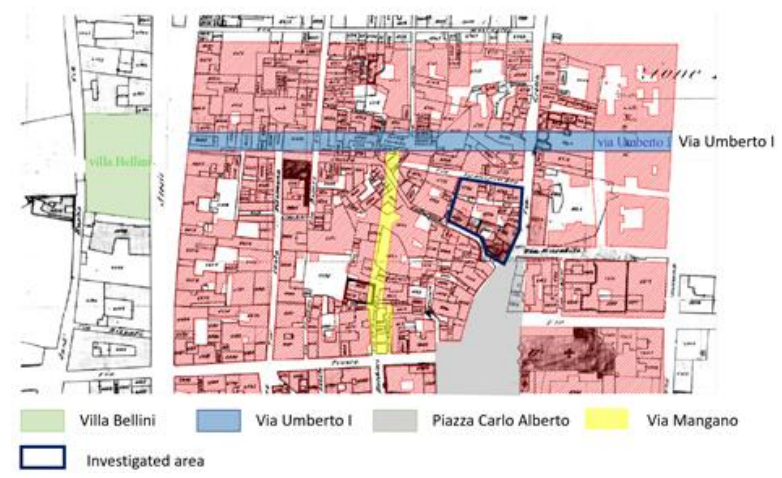

Figure 2. Overlapping between current urban fabric (in red) and the cadastral map of 1876 .

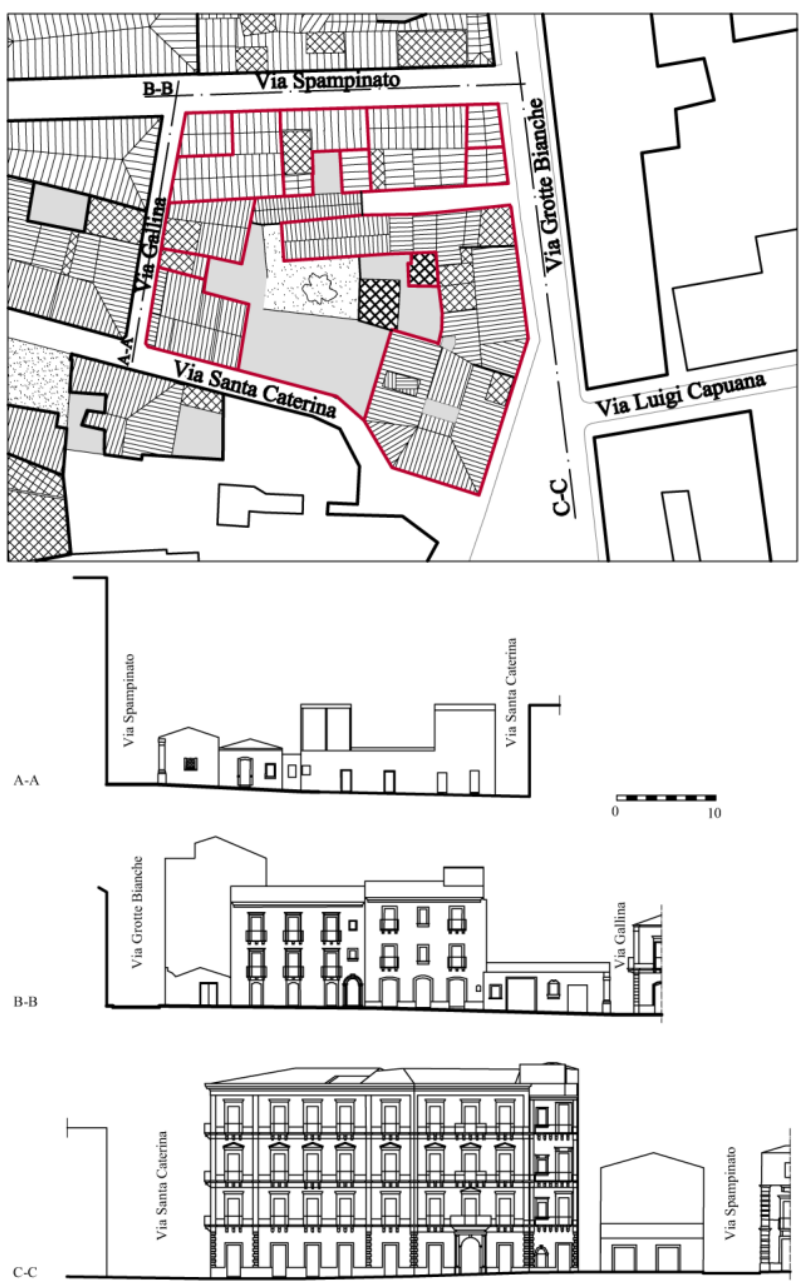

Figure 3. view of the investigated area and metric drawings along the streets which highlight the narrowness of the alleys and the different building typologies. 
The high level of complexity of the city block is due to the spontaneous evolution of various building typologies. The building pattern still largely retains the features of the Rinazzo district. This heritage is threatened by renovation operations that eliminate the typological and formal characteristics of the original buildings in the neighbourhood. The buildings, groundfloor houses or maximum two/three-storey houses, stand on enclosed and corner plots, with narrow, low fronts defined by simple corner stones and mostly covered by pitched roofs. Only in a few cases, such as the building at the end of the road between via Grotte Bianche/piazza Carlo Alberto/via Santa Caterina, there are multi-family buildings on several floors, with small courtyards from which the inner rooms are illuminated.

\section{EXPEDITIOUS URBAN DIGITAL SURVEY}

\subsection{Data acquisition \& processing}

In order to be able to compare the results from the two survey methodologies adopted, photogrammetry from $360^{\circ}$ images and iMMS via SLAM, a TLS-based survey has been used as a reference. BLK360 by Leica Geosystem has been used for SLAM scans. The scanner, which is particularly light and modest in size, permitted the acquisition of 20 scans along the entire perimeter of the block under severe conditions of vehicular and pedestrian traffic. In order to make the final point cloud more uniform, we used a high resolution acquisition only in the presence of considerable heights and open spaces. In this way, the on-site acquisition phase lasted less than 1 hour. The clouds were pre-aligned on-site via the Field360 app. This reduced the time needed for the cloud registration phase within Register360.

For the SFM survey, we used a GOPRO Fusion 360 action camera. The spherical photos were acquired on the move, holding the camera through the support pole at a height of about $2 \mathrm{~m}$ above the ground. We used the sequenced image mode, setting an interval of 2 seconds and leaving the shooting parameters in automatic. Walking at a normal pace around the block for about 200 meters (slowing down near the corners) we acquired 144 spherical photos with a resolution of 5760x 2880 pixels in 4 minutes and 44 seconds. During the acquisition phase the axes of the two lenses were kept perpendicular to the axis of the road in order to avoid excessive deformation of the photos near the fronts of the buildings (fig. 4). Through the software Fusion Studio, the 360 photos have been extracted after stitching operations between the front camera and the rear camera. In addition, images were post processed in relation to shadows, highlights and exposure with the aim to reduce radiometric differences due to lighting conditions. The result is a dataset of equirectangular images that we imported into Agisoft Metashape for the processing phase.

First, during the automatic alignment phase, all images were correctly aligned. Once this step was completed, we obtained a sparse cloud of 144,009 points using 'High' as level of accuracy (fig. 4). Afterwards, we reconstructed the dense cloud in 'High' quality and with the 'Moderate' depth filtering. The latter was preferred to the 'Aggressive' one to avoid relevant loss of data during the reconstruction. The final dense cloud consists of $43,422,818$ points. The entire pipeline, from acquisition to processing and cleaning phase, required about 3 hours and 15 minutes. The final weight of the point cloud is equal to $639 \mathrm{Mb}$.
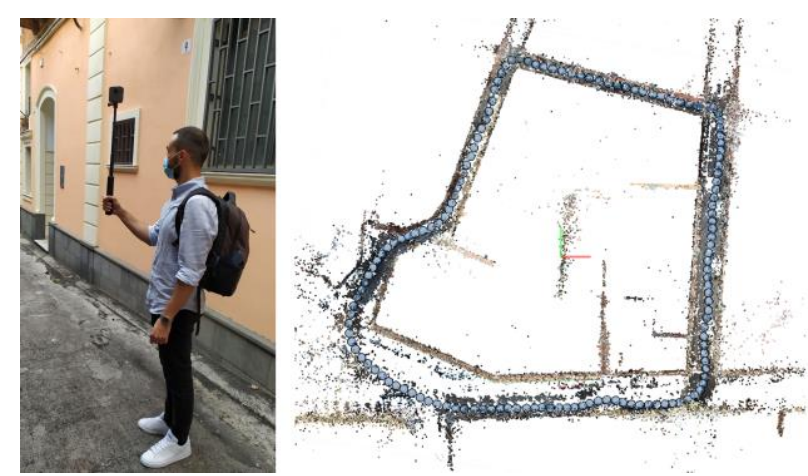

Figure 4. On the left: GOPRO Fusion 360 on site during acquisition. On the right: sparse cloud with points of acquisition inside Metashape.

Regarding the SLAM survey, we used the new BLK2GO sensor from Leica Geosystem. As already mentioned in paragraph 3, the characteristics of the sensor determine a specific procedure for using the instrument. In particular, great care must be taken during the initialisation phase of the sensor, since lifting it too suddenly from the ground or in the presence of heavy vehicular/pedestrian traffic may interfere with the start of the acquisition. The same path was covered as with the GOPRO Fusion except for obstacles caused by intense vehicular traffic. The perimeter of the block was covered in 7 minutes and 34 seconds. The operator was particularly careful when changing direction, decreasing the speed of the walk and rigidly rotating the torso in the chosen direction to avoid sudden movements during data acquisition. At this point, the raw data from the instrument was imported into Register360 and the noise reduction filter was applied. The resulting cloud consists of $13,477,837$ points. The entire data acquisition and processing operation took less than 30 minutes with a data size of $246 \mathrm{Mb}$ (figg. 5.6).
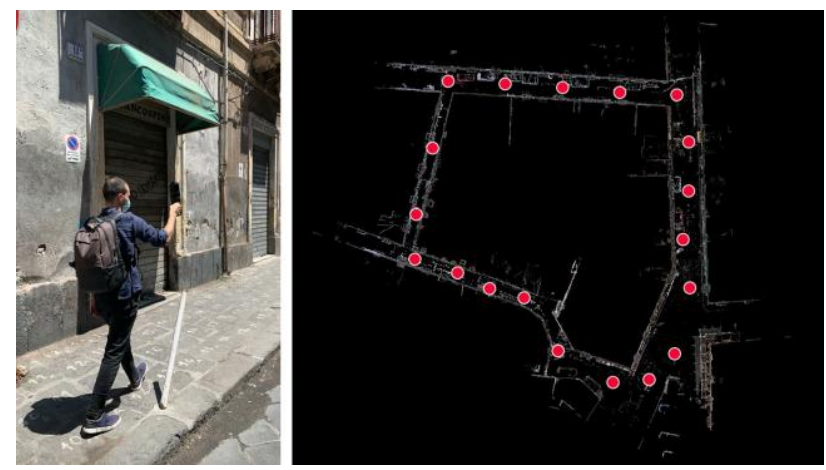

Figure 5. On the left: BLK2GO on site during acquisition. On the right: imported point cloud in Register 360.
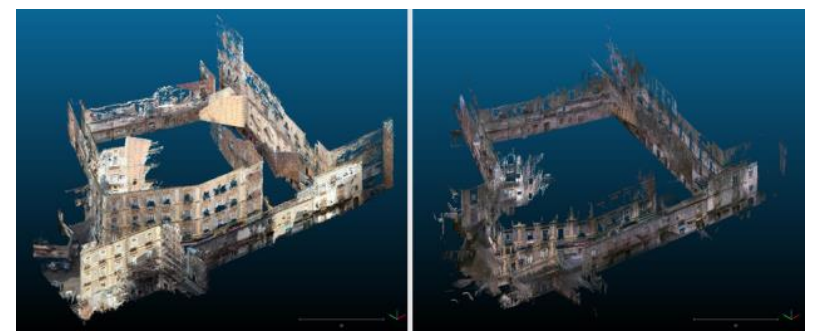

Figure 6. On the left: 360 SFM (GOPRO Fusion 360) cloud. On the right: SLAM (BLK2GO) cloud. 


\subsection{Accuracy evaluation}

The accuracy evaluation dealt with the geometric features of the point clouds produced and their ability to describe reality. In particular, we followed the approach proposed by Sammartano et al (2021) and Tucci et al. (2018). We conducted an analysis of the global accuracy of SFM and SLAM point clouds compared to TLS cloud. This was followed by an analysis of the local accuracy on a portion of the cloud representative of the building types present in the historical Italian urban centers. These two analyses are necessary because, as indicated in 2.3 section, over wide distances the point clouds produced by SLAM can be affected by drift errors while locally they closely reproduce the built environment. Afterwards, we carried out specific analyses on geometric characteristics such as: planarity, density, roughness and curvature of both point clouds. All the analyses were conducted using the algorithms provided by the opensource software CloudCompare (CC).

To assess the global accuracy, SLAM and SFM scans have been aligned with TLS scan which was used as reference. This was done by locating 15 points distributed along the perimeter in areas common to both SLAM and SFM data. For the global analysis, we preferred to use the $\mathrm{M} 3 \mathrm{C} 2$ algorithm. This algorithm allows a faster analysis than the traditional Cloud-toCloud (C2C) method and is less sensitive to noise. M3C2 initially calculates the evolution of the mean surface with respect to a scale that takes into account the roughness of the scan. It then evaluates its trend with respect to the normals calculated with respect to a significant point (in the case study, the barycentre was used) using a subset of points (corepoints) to speed up the analysis. Compared to $\mathrm{C} 2 \mathrm{C}$, it does not require the computation of a mesh and therefore reduces inaccuracy due to cloud roughness. The result of the analysis can be directly represented on the point cloud used as a reference (TLS). We therefore applied a colour scale to display the deviations in a range from $-30 \mathrm{~cm}$ to $+30 \mathrm{~cm}$. For the SFM scan analysis, a mean deviation of $3.3 \mathrm{~cm}$ and a higher standard deviation of $1.05 \mathrm{~m}$ were calculated.
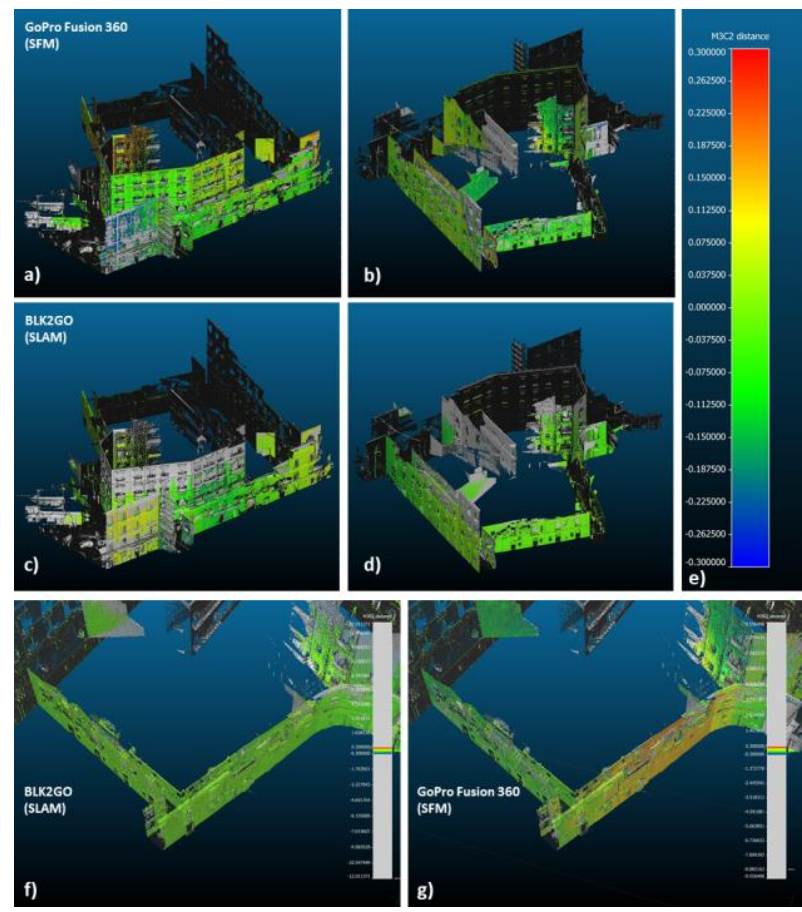

Figure 7. M3C2 global analyses of SFM cloud $(a, b)$ and SLAM cloud (c, d). The scalar field (e) ranges from blue (-30 $\mathrm{cm})$ to red $(+30 \mathrm{~cm})$. Focus on differences in narrow streets between SLAM (f) and 360 SFM (g).

From the visual analysis of the comparison, it is possible to notice higher deviation values near the ledges, in the noisiest areas and in the upper part of the facades $(22.5$ to $30 \mathrm{~cm})$. For the road, ground floor and facades planes, the deviation values are in the range of $+/-3 \mathrm{~cm}$. Regarding the analysis with the SLAM scan, the mean deviation is $1.1 \mathrm{~cm}$ while the standard deviation is $1.26 \mathrm{~m}$. In contrast to the SFM scan, the point cloud at the ledges has values that correspond to the mean deviation $(+/-1 \mathrm{~cm})$. The highest deviation values $(+/-20 \mathrm{~cm})$ mainly concern the ground floors (disadvantaged by heavy vehicular and pedestrian traffic). From the cloud it is visually possible to notice an absence of data beyond the first floor of the buildings and an increase in deviations with increasing elevation (fig. 7). In order to evaluate the accuracy at urban scale (definition of the investigated block perimeter) horizontal slices were extracted at ground level $(1.60 \mathrm{~m}$ from the lowest point of the cloud). These were then analysed using $\mathrm{C} 2 \mathrm{C}$. The main observation that emerges is that for the SFM scan the lowest offset values are found in the proximity of wider and well-lit urban canyons while the SLAM scan performs better in narrower and shaded urban canyons. It is worth highlighting that the SLAM scan has a drift that in the maximum deviation reaches $20 \mathrm{~cm}$ over a distance of about $200 \mathrm{~m}$ (fig. 8).

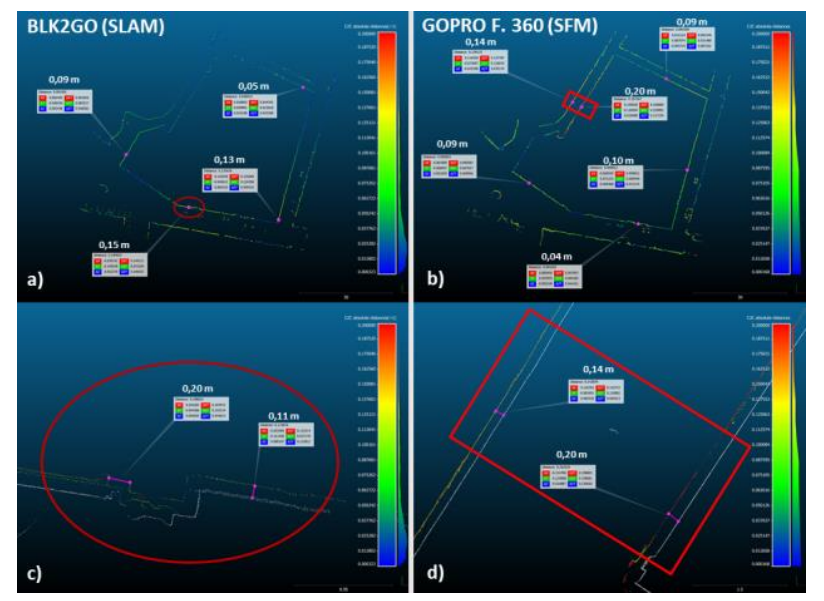

Figure 8. C2C analyses of horizontal slices for SLAM cloud (a, c) and 360 SFM cloud (b, d). Better viewed digitally.

For the local analysis, we chose a portion of the building on four levels that has architectural elements typical of Italian historic centers, with an exception for planarity analysis. In this case, we chose a plain wall (dimensions $3.50 \times 2.50$ ) at ground level.

With regard to the analysis of planarity, the SFM and SLAM scans are then evaluated using the Cloud-to-Mesh algorithm. The table below shows the percentages of points with respect to the ranges of $+/-1 \mathrm{~cm}$ and $+/-3 \mathrm{~cm}$ (tab. 2 ).

\begin{tabular}{|l|c|c|}
\hline \multicolumn{1}{|c|}{ Sensors Type } & $+/-1 \mathrm{~cm}$ & $+/-3 \mathrm{~cm}$ \\
\hline TLS & $94 \%$ & $100 \%$ \\
SFM (360 images) & $35 \%$ & $78 \%$ \\
SLAM & $92 \%$ & $96 \%$ \\
\hline
\end{tabular}

Table 2. Percentage of points matching the plane identified by the TLS scan in the given ranges.

For the density analysis, the Number of Neighbours algorithm was used, which returns the number of points contained within a sphere of specified radius. In this work we have used a sphere radius of $5 \mathrm{~cm}$. Visual analysis of the scans reveals a more homogeneous density in the SFM scan compared to the SLAM 
scan where there are more data gaps between overlapping parts (fig. 9). The table below (tab. 3) shows the mean deviations from the three scan types.

\begin{tabular}{|c|c|}
\hline Sensors Type & $\mathrm{N}^{\circ}$ of Neighbours $(\mathrm{r}=0.05 \mathrm{~m}) \mid$ Mean \\
\hline TLS & 268 \\
\hline SFM (360 images) & 50 \\
\hline BLK2GO & 16 \\
\hline
\end{tabular}

Table 3. Density analysis: mean deviations from the three scan types.
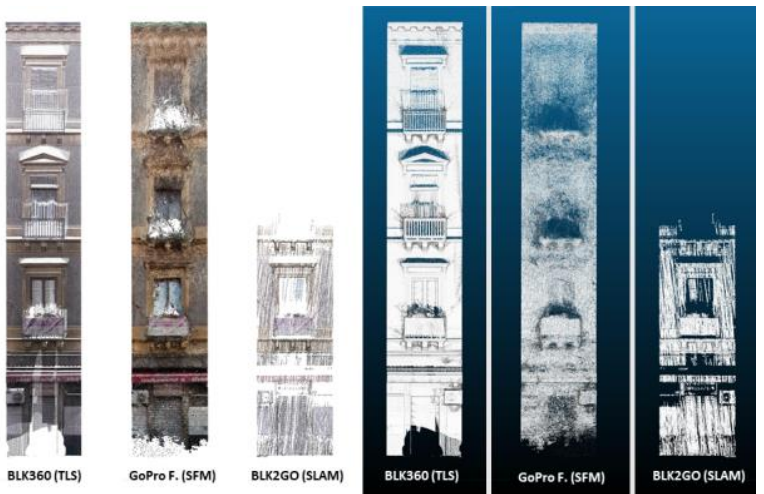

Figure 9. Density comparison between TLS, SFM and SLAM.

With reference to the roughness values of the scans, we considered a radius of $5 \mathrm{~cm}$ in this case as well. This comparison shows that the SLAM scan has relatively low noise values, with peaks at the overhangs. On the contrary, the SFM scan presents more noise and it is distributed over the whole cloud. Finally, the curvature was analysed in order to understand the level of detail achievable by the two clouds in relation to the recognition of openings in the facades. In the SLAM scan, edges can be clearly identified with $15 \mathrm{~cm}$ kernel sphere, while the radius has to be doubled in the SFM scan. However, the higher noise level makes it difficult to clearly identify edges in the SFM scan (fig. 10).

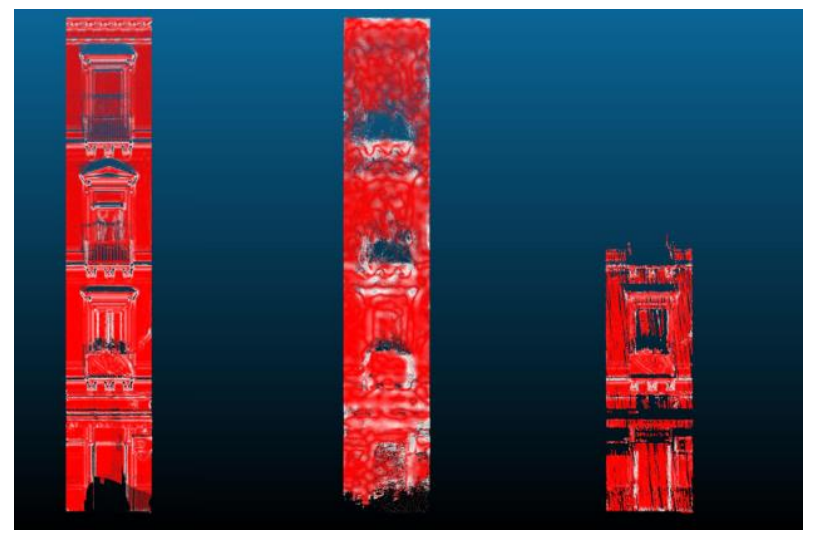

Figure 10. Curvature analysis between (from left to right) TLS, SFM, SLAM scans.

\section{CIM}

\subsection{From LOD 0 to LOD 2}

The pipeline of the CIM model starts with the use of geodata from datasets realised through research activities in the past. These geodata consist of a shapefile which, in addition to the building footprints, contains various metadata (geometric characteristics, construction period, references to historical documents, etc.) concerning each building (Galizia, Santagati, 2012). Through the Urban plugin, it was possible to read inside
Grasshopper (Rhinoceros VPL) both the geometries and the metadata contained in the shapefile. The initial modelling step was to achieve LOD 1 (boxes) in accordance with the standards defined by CityGML. Therefore, we extracted the information of the eave height of the façades in order to define the main volumes for the city block. If the height information was missing, the floor number was used, assuming a measurement of the inter-floor level that can be hypothesised on the basis of the known historical sources.

LOD 2 consists of a semantic decomposition of the surfaces that form the boxes. It also involves the definition of roof geometries. Using Grasshopper it was possible to filter the surfaces on the basis of their normals. The first filter made it possible to divide vertical and horizontal surfaces. Horizontal surfaces were further subdivided on the basis of their centroid. In this way, the ground floor and the base of the roof were identified. Through the number of elevations contained in the metadata we inserted the remaining intermediate floors for each building. At this point we created BIM objects for the building components through the VisualARQ plugin that allows us to insert them into Grasshopper. The BIM modelling covered the floor, the perimeter walls and the roof. In this way, the model will be ready to receive information that will be entered in the future as a result of specific investigations (fig. 11).

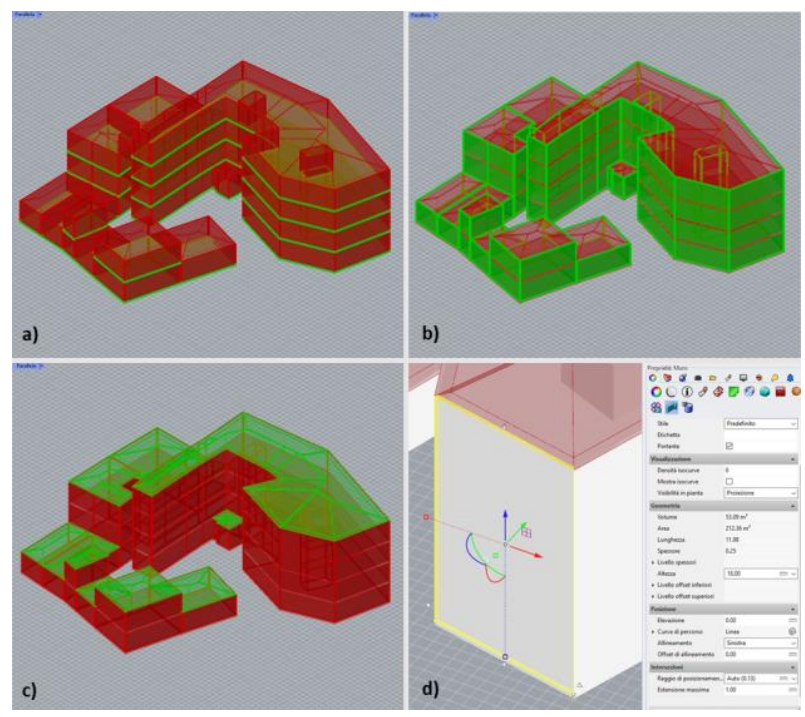

Figure 11. LOD 2 CIM Model in Grasshopper. Focus on slabs (a), walls (b), roofs (c) and BIM attributes with VisualARQ plugin for Rhinoceros (d).

\subsection{Towards LOD 3: SFM techniques and webdata}

LOD 3 according to CityGML standards consists of modelling the elements of the envelope, in particular the openings in the facades. In order to support the modelling operations, it is useful to include the point cloud of the block fronts obtained through SFM techniques. In this way it will be possible to identify the position of the openings with a metric error tolerance compatible with the scale of urban representation $(1: 200)$. The area studied in this research work does not easily permit the use of UAVs for the photogrammetric reconstruction of roof geometry. Moreover, the high level of complexity of the geometry of the roofs hinders the definition of procedural modelling processes for their correct representation. To fill this gap, we downloaded the 3D mesh of the block from Google Earth using the software RenderDoc and the add-on MapsModelsImporter for Blender. Despite the metric 
inaccuracy that affects these models, the quality of the mesh roofs is still sufficient to help the user in the manual urban modelling phase (fig. 12).

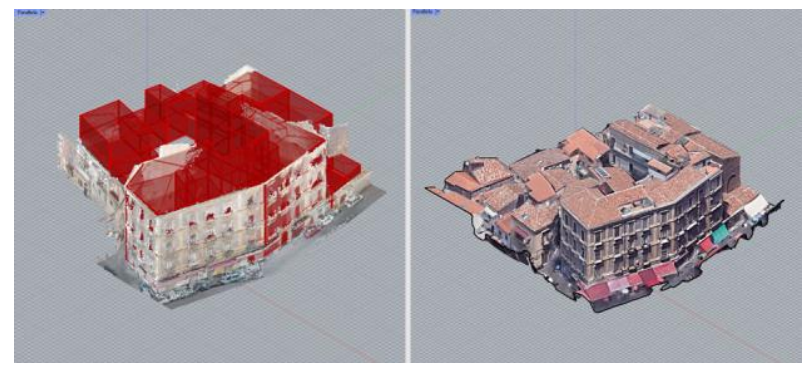

Figure 12. On the left, SFM point cloud in Grasshopper. On the right: 3D Google Earth mesh of the city block under examination. Model attribution: Google Earth, @2021 Google.

\section{RESULTS \& DISCUSSION}

The analyses conducted so far allow qualitative and quantitative comparisons between the two tested technologies. First of all, we need to keep in mind that the results have to be assessed against the finality of the work. In this case, a fast urban 3D acquisition for the creation of a CIM of the historical center to be updated during the time. The table below (tab. 4) compares the main characteristics that emerged from the technical and qualitative analyses in section 5 .

\begin{tabular}{|l|c|c|}
\hline \multicolumn{1}{|c|}{ Category of comparison } & $\begin{array}{c}\text { SFM (360 } \\
\text { images) }\end{array}$ & $\begin{array}{c}\text { SLAM } \\
\text { (iMMS) }\end{array}$ \\
\hline Main planes reconstruction & Medium & High \\
Elevation data acquisition & up to 3rd floor & up to 1st floor \\
Readability of RGB data & High & Medium \\
Level of geometric accuracy & Low & Medium \\
Metric quality & $1: 200$ & $1: 100 / 1: 200$ \\
Noise level & High & Low \\
Noise location & Diffuse & Edges \\
Density & High & Low \\
Max deviation (ca.) & $30 \mathrm{~cm}$ & $20 \mathrm{~cm}$ \\
Shift & $/$ & $20 \mathrm{~cm}$ \\
Data acquisition time & $4^{\prime} 48^{\prime}$, & $74^{\prime}$, \\
Data processing time & $3 \mathrm{~h} 25^{\prime}$ & $20 '$ \\
User expertise needed & Medium/High & Low \\
Price of the sensor (ca.) & $500 €$ & $50,000 €$ \\
\hline
\end{tabular}

Table 4. Comparison table between SFM (GOPRO Fusion 360) and SLAM (BLK2GO) workflows.

The comparison shows the potentialities of BLK2GO SLAM technology even though there are limitations. The main one is the acquisition range: $0.5-25$ metres according to the technical specifications and around 10 metres in this particular case study. On the other hand, the SFM technique with spherical images is sustainable with respect to the product it returns, despite the lower quality of the cloud compared to the SLAM scan and the problems related to light conditions. The BLK2GO appears to be useful in historic urban areas consisting of buildings on $1 / 2$ levels, for massive survey operations and with on-site personnel who may not be experienced. Possible applications could be urban post-emergency scenarios. The GOPRO is an effective, affordable tool but requires the involvement of experts. Therefore, we assume a more localised use related to risk assessment and prevention activities. With regard to the CIM workflow, high granularity of input geodata can be exploited to its full potential and implemented through the use of Grasshopper. The association of these survey data with CIM models can allow the definition of expeditious assessment protocols that support the evaluation and mitigation of urban and territorial risks. The use of existing geodata and webdata (e.g. Google Earth mesh) optimises the necessary resources, enabling even the smallest town administrations to undertake survey campaigns to assess the state of their historic centers.

\section{CONCLUSIONS}

In conclusion, this research was aimed to explore the potentialities of expeditious surveying techniques for the creation of informed and responsive 3D city models of historical centers. The workflow carried out contributed to evaluate the performances of cutting-edge technologies (iMMS based on SLAM and spherical photogrammetry) in comparison with well established TLS technologies. In particular, SLAM technology is currently in a phase of evolution and requires further studies in the urban field to fully understand its potential. Indeed, the expeditious urban survey is fundamental in relation to methodologies such as CIM in order to overcome the threshold of LOD 2 and allow accurate analysis and simulations for the urban scale.

The complexity of the investigated case study helped to point out criticalities and advantages for setting up expeditious protocols for urban survey. Finally, the critical-analytical reading of archive sources, the interpretation of historical cartography and iconography, the reading of the geometricdimensional data obtained from the instrumental survey of the fronts and the road sections of the block, the creation of the CIM ensured to understand the historical-morphological values of the building-environmental context.

\section{ACKNOWLEDGEMENTS}

Authors would like to thank Mr Nicola Di Blasi and Rosario Caruso from Leica Geosystem for the use of BLK2GO and on site support.

F.M. La Russa is author of par. 3, 5, 6 and 7, M. Galizia is author of par. 1, 4 and 8, C. Santagati is author of par. 1, 2, 3, 7 and 8 .

\section{REFERENCES}

Abate, D., Toschi, I., Sturdy-Colls, C., and Remondino, F.: A low-cost panoramic camera for the $3 \mathrm{~d}$ documentation of contaminated crime scenes, Int. Arch. Photogramm. Remote Sens. Spatial Inf. Sci., XLII-2/W8, 1-8, https://doi.org/10.5194/isprs-archives-XLII-2-W8-1-2017, 2017

Barazzetti, L., Previtali, M., , Roncoroni, F., 2018. Can we use low-cost 360 degree cameras to create accurate 3D models? Int. Arch. Photogramm. Remote Sens. Spatial Inf. Sci., 42, 69-75. https://doi.org/10.5194/isprs-archives-XLII-2-69-2018

Barrera.Vera, J. A., Benavides-Lopez, J.A., 2018, Handeld mobile mapping applied to historical urban areas. DISEGNARECON, 11 (21), 20.3-20.14.

https://doi.org/10.20365/disegnarecon.21.2018.20

Bocconcino, M., Turco, M., Vozzola, M., Rabbia, A., 2021. Intelligent information systems for the representation of the city - Urban survey and design for resilience. Sposito, C. (ed.) Possible and preferable scenarios of a sustainable future.Towards 2030 and Beyond, 90-107, Palermo University Press. DOI: 10.19229/978-88-5509-232-6/562021. 
Boido, C., Davico, P., Spallone, R., 2021. Digital Tools Aimed to Represent Urban Survey. In (ed) Khosrow-Pour, M. (ed) Encyclopedia of Information Science and Technology, 5, 11811195. Hershey: IGI Global DOI: 10.4018/978-1-7998-34793.ch082

Bonfanti, I., Colucci, E., De Ruvo, V., Del Giudice, M., Fasana, S., Iacono, E., Lingua, A.M., Matrone, F., Marino Ventura, G., Zerbinatti, M., 2021. Development of an integrated BIM-GIS management tool for maintenance plan of historical heritage. Proceedings of the joint international event 9th ARQUEOLÓGICA 2.0 \& 3rd GEORES, Valencia (Spain). 26-28 April 2021 pp 247-254, Editorial Universitat Politècnica de València. DOI https://doi.org/10.4995/Arqueologica9.2021.12131

Coppo, D., Boido, C., 2010. Rilievo Urbano, conoscenza e rappresentazione della città consolidata. Firenze: Alinea.

Chiabrando, F., Della Coletta, C., Sammartano, G., Spanò, A. T., \& Spreafico, A., 2018. "TORINO 1911" project: A contribution of a SLAM-based survey to extensive 3D heritage modeling. IInt. Arch. Photogramm. Remote Sens. Spatial Inf. Sci., 42, 225-234. https://doi.org/10.5194/isprs-archives-XLII2-225-2018

De la Plata, A.R.M.; Franco, P.A.C.; Franco, J.C.; Bravo, V.G., 2021. Protocol Development for Point Clouds, Triangulated Meshes and Parametric Model Acquisition and Integration in an HBIM Workflow for Change Control and Management in a UNESCO World Heritage Site. Sensors 2021, 21, 1083. DOI: https://doi.org/10.3390/s21041083

Galizia, M., Santagati, C., 2012. GIS is/and knowledge. Documenting and managing Catania's historic urban heritage, Journal of Civil Engineering and Architecture, vol. 6 (8), 1014-1020. DOI:10.17265/1934-7359/2012.08.009

Gentile Cusa, B., 1888. Piano regolatore pel risanamento e per l'ampliamento della città di Catania, Catania, Tipografia C. Galàtola.

Julin, A., Jaalama, K., Virtanen, J.-P., Pouke, M., Ylipulli, J., Vaaja, M., Hyyppä, J., 2018. Characterizing 3D City Modeling Projects: Towards a Harmonized Interoperable System. In ISPRS International Journal of Geo-Information, 7(2), 55. MDPI AG. DOI: http://dx.doi.org/10.3390/ijgi7020055

Liu, X., Wang, X., Wright, G., Cheng, J., Li, X., \& Liu, R., 2017. A State-of-the-Art Review on the Integration of Building Information Modeling (BIM) and Geographic Information System (GIS). In ISPRS International Journal of GeoInformation, 6(2), 53. MDPI AG. DOI: http://dx.doi.org/10.3390/ijgi6020053

Lu, W., Peng, Y., Xue, F., Chen, K., Niu, Y., \& Chen, X., 2018. The Fusion of GIS and Building Information Modeling for Big Data Analytics in Managing Development Sites. In Comprehensive Geographic Information Systems, pp. 345-359. DOI: 10.1016/B978-0-12-409548-9.09677-9

La Russa, F. M., Santagati, C., 2020. From OpenData to city models: an Antifragile approach for City Information Modeling. Dn - Building Information Modeling, Data \& Semantics, 7, 8395 .
Limongiello M., Ronchi, D., Albano V., 2020. BLK2GO for DTM generation in highly vegetated area for detecting and documenting archaeological earthwork anomalies. IMEKO TC4 International Conference on Metrology for Archaeology and Cultural Heritage Trento, Italy, October 22-24, 2020, 316-321.

Nocerino, E., Menna, F., Remondino, F., Toschi, I., \& Rodríguez-Gonzálvez, P., 2017. Investigation of indoor and outdoor performance of two portable mobile mapping systems. In F. Remondino \& M. R. Shortis (Eds.), Videometrics, Range Imaging, and Applications XIV, edited, SPIE 2017 (p. 103320I). https://doi.org/10.1117/12.2270761

Piniotis, G., Soile, S., Bourexis, F., Tsakiri, M., and Ioannidis, C., 2020. Experimental assessment of $3 d$ narrow space mapping technologies, Int. Arch. Photogramm. Remote Sens. Spatial Inf. Sci., XLIII-B2-2020, 149-156. https://doi.org/10.5194/isprs-archives-XLIII-B2-2020-1492020

Predari, G., Bartolomei, C., Morganti, C., Mochi, G., Gulli, R., 2019. Expeditious methods of urban survey for seismic vulnerability assessments. Int. Arch. Photogramm. Remote Sens. Spatial Inf. Sci., XLII-2/W17, 271-278.

https://doi.org/10.5194/isprs-archives-XLII-2-W17-271-2019

Restuccia, F., Galizia, M., Santagati, C., 2011. Knowledge and/is safeguard: Via Umberto and the Rinazzo quarter in Catania. In: C. Gambardella (ed.) Le vie dei Mercanti, S.A.V.E. Heritage. Safeguard of Architectural, Visual, Environmental Heritage. Collana Fabbrica della Conoscenza vol 10, 1-10, La Scuola di Pitagora editrice

Sammartano, G., Spanò, A., 2018. Point clouds by SLAMbased mobile mapping systems: accuracy and geometric content validation in multisensor survey and stand-alone acquisition. Applied Geomatics, 10(4), 317-339. https://doi.org/10.1007/s12518-018-0221-7

Sammartano, G., Previtali, M., Banfi, F., 2021. Parametric generation in HBIM workflows for slam-based data: discussing expectations on suitability and accuracy. Proceedings of the joint international event 9th ARQUEOLÓGICA 2.0 \& $3 \mathrm{rd}$ GEORES, Valencia (Spain). 26-28 April 2021, pp 374-388, Editorial Universitat Politècnica de València.

DOI: https://doi.org/10.4995/Arqueologica9.2021.12155

Salgues, H., Macher, H., and Landes, T.: Evaluation of Mobile Mapping Systems for Indoor Surveys, Int. Arch. Photogramm. Remote Sens. Spatial Inf. Sci., XLIV-4/W1-2020, 119-125, https://doi.org/10.5194/isprs-archives-XLIV-4-W1-2020-1192020, 2020.

Teppati Losè, L., Chiabrando, F., Novelli, F., Patrucco, G., Perri, S., 2021. Documentation of a complex cultural heritage asset with integrated geomatic survey: the Montanaro bell tower. Proceedings of the joint international event 9th ARQUEOLÓGICA 2.0 \& 3rd GEORES, Valencia (Spain). 2628 April 2021 pp 177-184, Editorial Universitat Politècnica de València DOI: https://doi.org/10.4995/Arqueologica9.2021.12107

Tucci, G., Visintini, D., Bonora, V., Parisi, E., 2018. Examination of Indoor Mobile Mapping Systems in a Diversified Internal/External Test Field. Applied Sciences, 8(3), 401. https://doi.org/10.3390/app8030401 